\title{
Empathie, mentalisation et comportements extério-risés chez les enfants d'âge scolaire hébergés en centre jeunesse : une étude exploratoire
}

\section{Empathy, mentalization and externalized behaviors in school-aged children in youth centers: An exploratory study}

\author{
Geneviève Dubé, Miguel M. Terradas, Olivier Didier, Rosalie Guillemette et \\ Julie Achim
}

Volume 48, numéro 2, 2019

URI : https://id.erudit.org/iderudit/1066147ar

DOI : https://doi.org/10.7202/1066147ar

Aller au sommaire du numéro

\section{Éditeur(s)}

Revue de Psychoéducation

ISSN

1713-1782 (imprimé)

2371-6053 (numérique)

Découvrir la revue

Citer cet article

Dubé, G., Terradas, M. M., Didier, O., Guillemette, R. \& Achim, J. (2019). Empathie, mentalisation et comportements extério-risés chez les enfants d'âge scolaire hébergés en centre jeunesse : une étude exploratoire. Revue de psychoéducation, 48(2), 347-371. https://doi.org/10.7202/1066147ar

\section{Résumé de l'article}

Les abus sexuels ou physiques, la négligence et les mauvais traitements psychologiques survenant au sein de la relation parent-enfant risquent d'entraver le développement des capacités d'empathie et de mentalisation (CM) chez l'enfant, capacités qui permettraient d'inhiber les comportements extériorisés. Cette étude exploratoire vise à documenter le niveau d'empathie et de mentalisation chez 16 enfants âgés entre 7 et 11 ans, hébergés en centre jeunesse, ainsi que les liens existants entre ces capacités et les comportements extériorisés. Des questionnaires évaluant l'empathie, les biais de mentalisation et les comportements extériorisés des jeunes ont été administrés aux éducateurs de suivi $(n=13)$ et aux enfants $(n=16)$, ces derniers ayant également participé à une entrevue permettant de qualifier leur CM. Les enfants présentent tous des comportements extériorisés et une capacité relativement faible d'empathie. Ils manifestent également un refus de mentaliser ou une absence de mentalisation, ainsi que peu de biais de mentalisation. Des liens significatifs entre l'empathie, la $C M$ et les biais de mentalisation ont été trouvés. Aucun lien entre ces variables et les comportements extériorisés n'a cependant été démontré. Les filles présentent des scores plus élevés que les garçons quant à la CM et à l'empathie. Aucune différence n'a été observée entre les enfants issus des différents services d'hébergement (unité de vie en internat ou foyer de groupe externe). Les résultats permettent de décrire les capacités d'empathie et de mentalisation des enfants hébergés en centre jeunesse, en plus de soutenir l'importance d'interventions basées sur ces facultés afin de tenir compte des déficits observés chez ces enfants.
Tous droits réservés @ La Corporation de la Revue Canadienne de Psycho-Éducation, 2019
Ce document est protégé par la loi sur le droit d'auteur. L’utilisation des services d’Érudit (y compris la reproduction) est assujettie à sa politique d'utilisation que vous pouvez consulter en ligne.

https://apropos.erudit.org/fr/usagers/politique-dutilisation/ 


\section{Empathie, mentalisation et comportements extério- risés chez les enfants d'âge scolaire hébergés en centre jeunesse : une étude exploratoire}

\section{Empathy, mentalization and externalized behaviors in school- aged children in youth centers: An exploratory study}

G. Dubé1

M. M. Terradas ${ }^{2}$

O. Didier ${ }^{2}$

R. Guillemette ${ }^{2}$

J. Achim²

1 Centre local de service communautaire (CLSC) de Villeray

2 Département de psychologie, Université de Sherbrooke

Cette recherche a été partiellement financée par le Fonds de recherche sur la société et la culture du Québec (FRQSC).

Correspondance :

Miguel M. Terradas

Département de psychologie, Université de Sherbrooke Campus de Longueuil 150, Place Charles LeMoyne, bureau 200 Longueuil (Québec) J4K 0A8.

Tél. : 450 463-1835, poste 61611 Téléc. : 450 463-6584

miguel.terradas@usherbrooke.ca
Résumé

Les abus sexuels ou physiques, la négligence et les mauvais traitements psychologiques survenant au sein de la relation parent-enfant risquent d'entraver le développement des capacités d'empathie et de mentalisation (CM) chez l'enfant, capacités qui permettraient d'inhiber les comportements extériorisés. Cette étude exploratoire vise à documenter le niveau d'empathie et de mentalisation chez 16 enfants âgés entre 7 et 11 ans, hébergés en centre jeunesse, ainsi que les liens existants entre ces capacités et les comportements extériorisés. Des questionnaires évaluant l'empathie, les biais de mentalisation et les comportements extériorisés des jeunes ont été administrés aux éducateurs de suivi $(n=13)$ et aux enfants $(n=16)$, ces derniers ayant également participé à une entrevue permettant de qualifier leur CM. Les enfants présentent tous des comportements extériorisés et une capacité relativement faible d'empathie. Ils manifestent également un refus de mentaliser ou une absence de mentalisation, ainsi que peu de biais de mentalisation. Des liens significatifs entre l'empathie, la $\mathrm{CM}$ et les biais de mentalisation ont été trouvés. Aucun lien entre ces variables et les comportements extériorisés n'a cependant été démontré. Les filles présentent des scores plus élevés que les garçons quant à la CM et à l'empathie. Aucune différence n'a été observée entre les enfants issus des différents services d'hébergement (unité de vie en internat ou foyer de groupe externe). Les résultats permettent de décrire les capacités d'empathie et de mentalisation des enfants hébergés en centre jeunesse, en plus de soutenir l'importance d'interventions basées sur ces facultés afin de tenir compte des déficits observés chez ces enfants.

Mots-clés: empathie, capacité de mentalisation, biais de mentalisation, comportements extériorisés, enfants. 


\begin{abstract}
Sexual or physical abuse, neglect and psychological abuse within the parent-child relationship may hinder the development of child's empathy and capacity to mentalize, abilities that contribute to inhibit externalized behaviors. This exploratory study aims to document the level of empathy and mentalizing capacity (MC) of 16 children aged between 7 and 11 years-old living in foster care in different residential services (residential unit or external group home), as well as the links between these abilities and externalized behaviors. Questionnaires assessing empathy, metalizing bias and externalized behaviors of these children were administered to their main child workers $(n=13)$ and the children $(n=16)$, who also participated in an interview to qualify their MC. All the children exhibit externalized behaviors, little mentalizing bias, refuse to mentalize or have no ability to do so, as well as a relatively low capacity for empathy. Significant links between empathy, MC and mentalizing bias were found. However, no link between these variables and externalized behaviors has been demonstrated. Girls have higher scores than boys on MC and empathy. No difference was observed between the children from the different residential services (residential unit or external group home). The results allow to describe the empathy and mentalizing abilities of children living in youth centers. They support the importance of considering interventions based on these faculties when working with these children.
\end{abstract}

\title{
Keywords: empathy, mentalizing capacity, mentalization bias, externalized beha- vior, children.
}

\section{Introduction}

Au Québec, 91 \% des signalements faits en 2017-2018 l'étaient pour des risques sérieux ou des motifs de négligence, d'abus physique ou sexuel, ou de mauvais traitements psychologiques, et $61 \%$ des enfants pris en charge par la Direction de la protection de la jeunesse (DPJ) étaient âgés de moins de 12 ans (Directeurs de la protection de la jeunesse [DPJ], 2018). Une prise en charge par la DPJ mène, dans la moitié des cas, à un retrait du milieu familial (DPJ, 2018). En 2017-2018, 10 \% des enfants ont d'ailleurs été confiés à des services de réadaptation en centre jeunesse (p. ex., unité de vie) ou à des ressources intermédiaires (p. ex., résidence de groupe externe). Les enfants hébergés en centre jeunesse qui bénéficient des services de réadaptation présentent des troubles graves de la conduite, d'importants problèmes émotionnels, ainsi que des difficultés sévères d'interaction avec le milieu familial, scolaire ou social. Plusieurs présentent également un problème de délinquance, ou ont des parents qui rencontrent d'importantes difficultés ou se montrent incapables d'exercer leurs rôles parentaux (Centre jeunesse de la Montérégie, 2017). Ce type d'hébergement est caractérisé par la présence d'autres enfants et d'éducateurs dans des unités de vie ou des foyers de groupe où sont proposés des activités et un encadrement adapté aux besoins et aux problèmes des jeunes (Centre jeunesse de la Montérégie, 2017). L'hébergement peut prendre la forme d'une unité de vie en internat ou d'une ressource intermédiaire, qui constitue un milieu plus ouvert où se retrouvent des enfants qui peuvent fonctionner dans un contexte communautaire (Centre jeunesse des Laurentides, 2017). Le placement en réadaptation est d'ailleurs considéré comme une mesure de dernier recours, notamment dans les situations où les enfants présentent des problèmes qui nuisent à leur développement ou à leurs interactions sociales (Geoffrion et Ouellet, 2013). 
Geoffrion et Ouellet (2013) ont réalisé une étude auprès de 586 éducateurs travaillant dans un service de réadaptation dans l'un des dix centres jeunesse de la province de Québec afin d'examiner les facteurs de risque qui prédisent la violence physique des jeunes à leur égard. Ils rapportent que 53,9\% des éducateurs indiquent avoir été victimes de violence physique au cours de la dernière année. Comparativement à ceux qui travaillent avec des adolescents, les éducateurs qui œuvrent auprès des enfants sont presque 15 fois plus à risque d'être victimes de violence physique (Geoffrion et Ouellet, 2013). Ainsi, il s'avère que le problème des comportements extériorisés manifestés par les enfants hébergés en centre jeunesse est inquiétant. Dans ce contexte, il semble à la fois pertinent et nécessaire de déterminer ce qui peut expliquer cette prépondérance de comportements extériorisés manifestés par ces enfants.

\section{Comportements extériorisés chez les enfants en contexte de protection de l'enfance}

Les troubles de comportements extériorisés réfèrent aux comportements de l'ordre de l'agir, c'est-à-dire aux comportements agressifs, oppositionnels et délinquants (Achenbach et Rescorla, 2001). La notion d'agir réfère à la tendance à recourir à des expressions comportementales qui ne sont pas médiées par un processus réflexif. Ces troubles se distinguent des troubles de comportements intériorisés, considérés moins manifestes, tels que les symptômes dépressifs, anxieux et somatiques (Achenbach et Rescorla, 2001).

Les enfants victimes de maltraitance ou de négligence, et parfois retirés de leur milieu familial, présentent des caractéristiques qui les distinguent des enfants issus de la population générale. Diverses études menées auprès d'eux suggèrent notamment qu'ils démontrent plus de problèmes extériorisés (Finzi, Ram, Har-Even, Shnit et Weizman, 2001; Hukkanen, Sourander, Bergroth et Piha, 1999; Lawrence, Carlson et Egeland, 2006; Nadeau, Nolin et Chartrand, 2013; Petrenko, Friend, Garrido, Taussig et Culhane, 2012) et de difficultés sociales et interpersonnelles (Camras, Grow et Ribordy, 1983; Luke et Banerjee, 2012; Tarren-Sweeney, 2013). Une étude canadienne s'intéressant aux problèmes de fonctionnement (p. ex., problèmes émotionnels, comportements extériorisés, difficultés au plan développemental) selon les catégories de mauvais traitements suggère d'ailleurs qu'entre 36 et $49 \%$ des enfants victimes de violence physique, d'abus sexuel, de négligence et de violence psychologique démontrent des comportements extériorisés (Lefebvre, 2012).

\section{Hypothèses de compréhension de l'expression des comportements extériorisés chez les jeunes en contexte de protection de l'enfance}

Les fonctions psychologiques se développant normalement au sein d'une relation d'attachement sécurisante risquent d'être affectées par l'expérience de mauvais traitements, s'ajoutant ainsi aux effets de la séparation des figures parentales lorsque ces enfants sont retirés de leur famille d'origine. Ces événements ayant eu lieu dans le cadre de la relation parent-enfant risquent de provoquer des traumas relationnels précoces, qui peuvent entrainer des difficultés au niveau de la régulation des affects (Courtois, 2004). Les études s'intéressant aux problèmes 


\section{0}

extériorisés présentés par les enfants victimes de négligence ou de maltraitance s'attardent particulièrement aux capacités d'identification, de compréhension et de régulation des affects, chez soi et chez l'autre, pour expliquer leurs difficultés (Camras et al., 1983; Healey et Fisher, 2011; Pears et Fisher, 2005). Ainsi, il est probable que les capacités d'empathie et de mentalisation de ces enfants soient affectées, ce qui pourrait expliquer, du moins en partie, leur recours à l'agir ${ }^{1}$.

\section{Empathie}

Définition. Les définitions de l'empathie sont nombreuses et proviennent de divers champs épistémologiques. Toutefois, la plupart convergent vers l'idée selon laquelle elle représente la capacité d'un individu à comprendre et à s'accorder à l'émotion d'une autre personne, sans qu'une confusion sur l'origine des émotions s'installe (Decety et Jackson, 2006; de Vignemont et Singer, 2006; Houzel, 2000). Deux composantes essentielles peuvent être mises de l'avant, soit l'empathie affective et l'empathie cognitive. L'empathie affective réfère à la capacité d'un individu de partager l'expérience émotionnelle d'autrui (Baron-Cohen et Wheelwright, 2004; Decety et Jackson, 2004), ce qui signifie que l'état affectif de l'autre suscite une identification partielle, provoquant chez soi une réaction émotionnelle, généralement de moins grande intensité, mais accordée à l'émotion de l'autre personne (Greenson, 1977). L'empathie cognitive, quant à elle, réfère à la capacité de se représenter l'expérience affective d'autrui, c'est-à-dire à interpréter les causes de l'émotion perçue (Decety et Jackson, 2004; de Wied, Gispen-de Wied et van Boxtel, 2010).

Empathie et comportements extériorisés. Une méta-analyse réalisée par Miller et Eisenberg (1988) indique que l'empathie est négativement reliée aux comportements extériorisés et antisociaux, ainsi qu'à l'agressivité, tant chez les garçons que chez les filles. Les enfants victimes d'abus physique démontreraient également moins d'empathie que les enfants n'ayant pas été abusés. Toutefois, les analyses, réalisées à partir des résultats d'études antérieures, suggèrent un soutien modeste et non consistant à l'hypothèse selon laquelle l'empathie peut être considérée comme un inhibiteur de l'agressivité. En 2007, Lovett et Sheffield effectuent une nouvelle recension critique de la documentation scientifique portant sur l'empathie et l'agressivité chez les enfants et les adolescents. Ils suggèrent que chez les enfants, le lien entre l'empathie affective et l'agressivité n'est pas démontré, les résultats étant trop variables entre les études pour qu'une conclusion puisse être tirée. Toutefois, la corrélation négative entre l'empathie affective et l'agressivité serait plus robuste auprès d'une population adolescente. Les différentes méthodes (p. ex., instruments choisis, mesures auto-rapportées ou comportementales, opérationnalisation des comportements agressifs) et les populations étudiées ( $p$. ex., âge des participants, variété des comportements agressifs) pourraient expliquer l'inconstance des résultats.

1. Une recension des écrits et des élaborations théoriques concernant les liens entre l'empathie, la $\mathrm{CM}$ et les comportements extériorisés chez les jeunes en contexte de Protection de l'enfance sont présentées dans : Dubé, G., Terradas, M. M., \& Domon-Archambault, V. (2018). Empathie, mentalisation et traumas complexes : quels liens avec les comportements extériorisés des enfants en protection de l'enfance? Enfance, 2, 343-372. 
Au Québec, une étude réalisée par Girard, Terradas et Matte-Gagné (2014) auprès de 51 garçons âgés entre 7 et 12 ans, issus d'écoles primaires régulières et présentant des troubles de comportement, a utilisé deux questionnaires mesurant l'empathie, l'un complété par les enfants et l'autre par leurs parents. Afin d'obtenir une seule mesure d'empathie par participant, la moyenne des scores globaux standardisés des deux instruments a été utilisée. Les résultats suggèrent que la capacité d'empathie est négativement associée à la présence de troubles de comportement.

\section{Empathie et comportements extériorisés chez les enfants ayant vécu} de la maltraitance. En 1995, une étude longitudinale de Rogosh, Cicchetti et Aber, réalisée aux États-Unis, auprès de 89 enfants (60\% de filles) avait comme objectif d'évaluer la capacité des enfants à comprendre les émotions des autres, une notion qui s'apparente à l'empathie, ainsi que leurs compétences sociales et la présence de comportements agressifs. L'échantillon était composé de deux groupes, soit un groupe clinique, formé de 46 enfants ayant vécu une ou plusieurs formes de maltraitance (négligence : $80 \%$, mauvais traitements psychologiques : $85 \%$, abus physique : $54 \%$, abus sexuel : $4 \%$, abus combinés : $83 \%$ ), alors que le groupe de contrôle était composé de 43 enfants issus de la population générale n'ayant pas été exposés à des situations de maltraitance. Les enfants ont été évalués à l'âge de 5,6 et 8 ans quant à leurs capacités à comprendre les émotions des autres. Des évaluations de leurs compétences sociales et de leurs comportements agressifs ont été réalisées par leurs enseignants et leurs camarades d'école. Les résultats suggèrent que les enfants maltraités présentent largement plus de difficulté à comprendre les émotions négatives d'autrui, moins de compétences sociales et de plus hauts niveaux de comportements agressifs que les enfants qui n'ont pas été exposés à ce type de situation (Rogosch et al., 1995).

Deux autres recherches, réalisées aux États-Unis, ont également exploré l'empathie chez les enfants et les adolescents maltraités. La première étude a été effectuée par Pears et Fisher (2005) auprès de 91 enfants âgés de 4 à 5 ans. L'échantillon était composé de 31 enfants non maltraités, résidants dans leur famille, et de 60 enfants hébergés en contexte de protection de l'enfance. Parmi ces derniers, $66 \%$ avaient subi de la négligence, $15 \%$ de l'abus sexuel, $15 \%$ de l'abus physique et $3 \%$ de la violence psychologique. Les résultats soulignent la présence de corrélations significatives entre la maltraitance, la situation d'hébergement et la difficulté à comprendre les émotions d'autrui. Toutefois, la surreprésentation d'enfants ayant vécu de la négligence dans le groupe d'enfants hébergés n'a pas permis aux chercheurs de départager l'influence d'autres formes de maltraitance sur l'empathie. La seconde étude a été effectuée par LeSure-Lester (2000) auprès de 40 adolescents ayant subi des abus ( $15 \%$ de filles; Mâge $=14,04$ ans pour les garçons; $M_{\text {âge }}=13,75$ ans pour les filles) et vivant dans un foyer de groupe. Les résultats montrent une forte corrélation positive entre l'empathie et la réduction des comportements agressifs envers les pairs $(r=0,57 ; p<0,0001)$ et le personnel $(r=0,59 ; p<0,0001)$. Une forte corrélation positive est également trouvée entre l'empathie et le respect des règles du foyer de groupe $(r=0,67 ; p<0,0001)$ chez les adolescents hébergés. Ainsi, un haut niveau d'empathie était significativement associé à moins d'agressivité interpersonnelle envers les pairs et le personnel. 
Toutefois, l'absence d'un groupe de contrôle composé d'adolescents non hébergés n'a pas permis de comparer les résultats avec ceux issus de la population générale.

Les résultats de ces études soutiennent donc l'existence d'une corrélation négative entre le niveau d'empathie des enfants et les comportements extériorisés. De même, dans une élaboration théorique basée sur ses expériences cliniques et des résultats d'études empiriques, Music (2011) pose l'hypothèse d'un déficit d'empathie associé aux expériences traumatiques dans l'enfance. Ainsi, il est justifié de penser que l'expérience de maltraitance ou de négligence dans l'enfance affecterait le développement d'un ensemble de capacités, dont l'empathie et la mentalisation.

\section{Mentalisation}

Définition. La mentalisation, telle que conceptualisée par Fonagy et Target (1996, 1997, 2000, 2007; Target et Fonagy, 1996), concerne la capacité de penser ses actions et celles d'autrui et d'y attribuer un sens. Elle se définit par la faculté d'un individu de percevoir et d'interpréter ses comportements et ceux des autres en termes d'états mentaux, c'est-à-dire en tenant compte des pensées, des désirs, des croyances, des sentiments, des attentes et des intentions reliés aux paroles ou aux gestes exprimés (Allen, Fonagy et Bateman, 2008). Cette notion s'inscrit à la fois aux niveaux personnel et interpersonnel, puisqu'elle permet d'interpréter ses comportements et ceux des autres, ainsi qu'aux niveaux cognitif et affectif, puisqu'elle implique de reconnaitre les états mentaux et de faire des hypothèses sur la façon dont ils influencent les comportements. Selon Allen et al. (2008), la capacité de mentalisation (CM) permet de moduler les émotions et de les rendre plus tolérables, puisqu'elle donne un sens aux comportements par le biais des représentations. En effet, mentaliser facilite l'identification, la tolérance, la régulation et l'expression des émotions, puisque l'individu cherche alors à en comprendre l'origine et la signification. II peut ensuite agir de manière cohérente avec son émotion ou saisir chez l'autre ce qui motive son comportement. Les relations interpersonnelles peuvent alors être perçues par l'enfant comme compréhensibles, prévisibles et porteuses d'intentions et de sens (Allen et al., 2008).

Mentalisation et comportements extériorisés. Peu de recherches ont porté sur les liens entre les difficultés de mentalisation et la présence de comportements extériorisés. Ceci peut s'expliquer en partie par le peu d'instruments de mesure de la $\mathrm{CM}$, notamment adaptés pour les enfants. En se basant sur leurs expériences cliniques et leurs travaux de recherche en contexte de protection de l'enfance, Achim et Terradas (2015) et Domon-Archambault et Terradas (2015) suggèrent toutefois que des déficits au niveau de la CM sont souvent observés chez les enfants qui consultent en pédopsychiatrie ou qui sont hébergés en centre jeunesse, et que ces enfants présentent un fonctionnement psychologique basé sur l'agir plutôt que sur la symbolisation. Fonagy, Bateman et Luyten (2012) soutiennent d'ailleurs que les enfants dont la $\mathrm{CM}$ n'est pas bien intégrée tendent à extérioriser leurs états mentaux plutôt qu'à se les représenter. Sutton, Reeves et Keogh (2000), dans leur étude menée en Angleterre auprès de 81 enfants âgés entre 11 et 13 ans, rapportent que certains enfants qui présentent des comportements extériorisés parviennent à attribuer des états mentaux aux autres, mais qu'ils en font un 
mauvais usage. Comme l'indiquent Sharp et Venta (2012), il peut ainsi s'agir de pseudo-mentalisation, c'est-à-dire d'une forme de mentalisation utilitaire, qui vise à manipuler ou contrôler l'autre, et qui ne témoigne pas d'une curiosité, d'un intérêt et d'un respect pour le monde interne d'autrui. Ainsi, ces enfants présenteraient tout de même un déficit au niveau de leur CM.

Mentalisation et comportements extériorisés chez les enfants ayant vécu de la maltraitance. À notre connaissance, Ensink et al. (2014) ont réalisé la seule étude québécoise mesurant la CM d'enfants ayant subi un abus sexuel en utilisant le Child Attachment Interview (Target, Fonagy, Shmueli-Goetz, Datta et Schneider, 1998). Les résultats de leur recherche, menée auprès de 46 dyades mère-enfant, dont l'enfant avait vécu un abus sexuel intrafamilial ( $n=22 ; 68,18 \%$ de filles; $M$ âge $=9,25$ ans) ou extrafamilial ( $n=24 ; 79,16 \%$ de filles; Mâge $=10$ ans), et d'un groupe de contrôle de 48 dyades (60,42 \% de filles; Mâge = 9,92 ans), montrent que les enfants ayant un historique d'abus sexuel (intrafamilial ou extrafamilial) possèdent une CM significativement inférieure à celle d'enfants du groupe de contrôle.

Ostler, Bahar et Jessee (2010), dans leur étude menée aux États-Unis auprès de 26 enfants hébergés en famille d'accueil $(42,31 \%$ de filles; Mâge $=9,4$ ans), ont eu recours à une entrevue semi-structurée (My Family Stories Interview; MFSI) et à un système de cotation de la mentalisation (Children's Mentalizing Scale; CMS) créés pour les besoins de leur recherche. Ainsi, bien que leurs instruments n'aient pas été validés, leurs résultats montrent que les enfants qui ont une bonne CM présentent moins de symptômes intériorisés et extériorisés.

Deux études réalisées auprès d'adolescents issus de la population générale ont également permis de mesurer la $\mathrm{CM}$ en ayant recours à l'Adult Attachment Interview (George, Kaplan et Main, 1996). La première, réalisée par Taubner et Curth (2013) en Allemagne auprès de 97 adolescents (48 \% de filles; Mâge $=15,94$ ans), montre une corrélation positive $(r=0,27 ; p<0,01)$ entre la présence d'abus (physique ou sexuel) durant l'enfance et des comportements agressifs à l'adolescence, de même qu'une corrélation négative $(r=-0,20 ; p<0,05)$ entre la présence d'abus et la CM. La CM aurait d'ailleurs un rôle médiateur dans la relation entre l'abus et les comportements agressifs. L'autre étude, menée par Taubner, White, Zimmerman, Fonagy et Nolte (2013) auprès de 104 adolescents $(43,27 \%$ de filles; Mâge $=16$ ans $)$, montre une corrélation négative entre la CM et les comportements agressifs proactifs $(r=-0,36 ; p<0,001)$ et réactifs $(r=-0,22 ; p$ $<0,05)$.

Mentalisation et biais de mentalisation. Sharp et Venta (2012) suggèrent que les enfants qui présentent des comportements extériorisés font preuve d'une mentalisation déformée (distorted mentalization), c'est-à-dire qu'ils présentent des biais de mentalisation. Ces biais peuvent être compris comme des styles de mentalisation qui s'appliquent davantage à la façon dont la personne perçoit les autres et elle-même. Ainsi, un individu peut mentaliser de façon rationnelle ou neutre, ou alors de façon trop positive ou trop négative (Sharp, Croudace et Goodyer, 2007). Sharp (2006) propose que les enfants qui présentent des traits antisociaux sont aux prises avec des distorsions cognitives négatives lorsqu'ils doivent interpréter les 
comportements d'autrui, alors qu'ils font preuve de distorsions positives lorsqu'ils interprètent leurs propres comportements. À notre connaissance, le seul instrument développé à cette fin et disponible actuellement (Social Stories Test; Sharp et al., 2007) permet seulement de mesurer les distorsions attribuées à autrui dans un contexte où les comportements à interpréter sont ceux de l'enfant lui-même (p. ex., on demande à l'enfant ce que les autres peuvent penser de lui dans une situation précise), et non pas la façon dont l'enfant interprète les comportements des autres. Par conséquent, l'instrument ne permet pas de faire la distinction entre les biais de mentalisation liés à soi ou à autrui. L'enfant ayant un biais de mentalisation positif peut tendre à affirmer que les autres le perçoivent d'une façon globalement positive et ainsi surestimer ses compétences. À l'inverse, un enfant présentant un biais de mentalisation négatif peut entretenir une vision négative et dévalorisante de la façon dont les autres le perçoivent.

Bien que les études s'intéressant aux biais de mentalisation soient peu nombreuses, deux d'entre elles, menées auprès du même échantillon issu de la population générale en Angleterre, suggèrent que les enfants qui adoptent des biais de mentalisation positifs sont plus susceptibles de présenter des symptômes extériorisés (Ha, Sharp et Goodyer, 2011; Sharp et al., 2007). Toutefois, une étude réalisée au Québec par Girard et al. (2014), utilisant le même instrument de mesure auprès d'enfants d'âge scolaire présentant des troubles de comportement, montre plutôt que ce sont les biais de mentalisation négatifs qui sont associés aux comportements extériorisés. Toutefois, l'utilisation des scores obtenus à l'instrument de mesure des biais de mentalisation diffère dans les deux études, ce qui pourrait expliquer les différences observées. En effet, Ha et al. (2011) et Sharp et al. (2007) ont réparti les enfants dans des catégories mutuellement exclusives selon le type de biais le plus fréquemment présenté, alors que Girard et al. ont calculé le nombre de biais de mentalisation positifs et négatifs présentés par chaque enfant. Cependant, les résultats montrent la présence de biais de mentalisation et vont ainsi dans le sens des études qui suggèrent que les enfants qui présentent des comportements agressifs tendent à attribuer des intentions hostiles aux autres dans les situations ambiguës, ce qui pourrait expliquer leurs comportements dans leurs interactions sociales (Akhtar et Bradley, 1991; Dodge, 1980).

Ainsi, certaines études ont mis en lumière que la CM ainsi que les biais de mentalisation sont liés aux comportements extériorisés. Toutefois, comme des études portant sur l'empathie ont également révélé de tels liens, il devient pertinent d'évaluer et de comparer ces deux variables au sein d'un même échantillon d'enfants en protection de l'enfance afin de départager leurs influences respectives sur les manifestations comportementales extériorisées.

\section{Objectifs}

La présente étude, à visée exploratoire, a d'abord pour objectif de documenter et de qualifier, chez des enfants québécois hébergés en service de réadaptation en centre jeunesse, la capacité d'empathie (globale, cognitive et affective), la CM et les biais de mentalisation (positifs, négatifs, neutres), ainsi que la présence de comportements extériorisés. Ensuite, l'étude vise à explorer les liens entre la CM, les biais de mentalisation, l'empathie et les comportements 
extériorisés, ainsi qu'à préciser les différences observées entre les enfants selon leur sexe et leur service d'hébergement (unité de vie en internat ou foyer de groupe externe).

\section{Méthode}

\section{Participants et procédure}

La présente étude s'inscrit dans une recherche plus large menée conjointement avec un centre jeunesse. Seize enfants ( 5 filles; Mâge $=9,31 ; E T=$ 1,54 ) âgés entre 7 et 11 ans constituent l'échantillon. Ils proviennent de deux unités de réadaptation : une unité de vie en internat $(n=8)$ et un foyer de groupe externe $(n=8)$. Treize éducateurs de suivi ont également pris part à l'étude. Leur participation a été sollicitée puisqu'ils côtoient les enfants au quotidien, alors que les parents de ces derniers présentent souvent des difficultés sur le plan de la santé mentale, vivent dans des conditions précaires et instables, les rendant difficiles à rejoindre, et qu'ils n'ont parfois pas eu de contact avec leur enfant depuis une longue période de temps. Certains éducateurs de suivi ayant deux enfants à leur charge, leur nombre est légèrement inférieur à celui du nombre d'enfants participant à l'étude.

Les approbations éthiques nécessaires ont été obtenues préalablement à la collecte de données. Les deux équipes d'éducateurs invitées à participer à l'étude ont d'abord été rencontrées par le responsable du projet et les assistantes de recherche afin de leur présenter les objectifs de l'étude, ainsi que le processus de collecte de données. Les éducateurs ayant consenti à participer ont signé le formulaire d'information et de consentement (FIC), puis présenté le projet de recherche aux parents des enfants et obtenu leurs consentements écrits. Une section du FIC à l'intention du parent était adressée directement aux enfants, leur expliquant, dans un langage adapté à leur âge, la nature de leur participation. Tous les enfants dont les parents ont consenti à leur participation ont pris part à la recherche.

Trois séances d'évaluation, variant entre 40 et 120 minutes, ont eu lieu avec chacun des enfants aux locaux du centre jeunesse. Divers instruments de mesure ont été administrés au cours des rencontres. La passation de l'Entrevue de l'attachement de l'enfant a été enregistrée sur support vidéo et audio afin de faciliter l'analyse et la cotation. Durant l'administration des questionnaires, tous les énoncés ont été lus à voix haute, par l'enfant ou par l'assistante de recherche, afin de s'assurer de la compréhension des participants et de la complétion de tous les énoncés. Des questionnaires ont été remis aux éducateurs lors de la première rencontre d'évaluation avec les enfants. À tout moment, il était possible pour le jeune de rejoindre son éducateur et à sa demande, son éducateur pouvait être présent dans le local durant les séances d'évaluation. 


\section{Questionnaires complétés par les éducateurs.}

Liste de vérification du comportement des jeunes de 6 à 18 ans (Child Behavior Checklist; CBCL; Achenbach et Rescorla, 2001). Ce questionnaire est composé de 113 énoncés mesurés par une échelle de Likert de 0 à 2 qui permettent d'explorer un vaste spectre de difficultés émotionnelles et comportementales chez les enfants (p. ex., anxiété, dépression, problèmes sociaux, comportements agressifs). II est fréquemment employé en clinique et en recherche et permet notamment de différencier les comportements intériorisés et extériorisés. L'instrument démontre une excellente cohérence interne et l'alpha de Cronbach pour les échelles de symptômes est de 0,95 . La fidélité inter-juges est de 0,96 pour les items mesurant les problèmes spécifiques et la fidélité test-retest (une semaine d'intervalle) varie entre 0,95 et 1,00 (Achenbach et Rescorla, 2001).

Mesure d'empathie de Griffith (GEM; Dadds et al., 2008)2. Le questionnaire, dans sa version française (Girard, Terradas et Achim, 2012a), est composé de 23 énoncés mesurés par une échelle de Likert de -4 à 4 et dont les résultats se divisent en trois scores : empathie cognitive, empathie affective et empathie globale. L'instrument est complété par l'éducateur de suivi pour rendre compte des capacités d'empathie qu'il perçoit chez l'enfant. Dans sa version originale anglaise, l'instrument démontre une bonne convergence avec des questionnaires d'empathie complétés par les enfants. Le GEM possède une bonne fidélité testretest $(r=0,91$ après une semaine; $r=0,69$ après six mois) et une bonne cohérence interne ( $\alpha=0,81$ pour l'empathie globale; $\alpha=0,62$ pour l'empathie cognitive; $\alpha=$ 0,83 pour l'empathie affective) (Dadds et al., 2008). L'indice de cohérence interne obtenu dans le cadre de la présente recherche est satisfaisant $(\alpha=0,78)$.

\section{Questionnaires et entrevue complétés par les enfants.}

Échelle d'empathie élémentaire (D'Ambrosio, Olivier, Didon et Besche, 2009). Ce questionnaire, composé de 20 énoncés mesurés par une échelle Likert variant de 0 à 4 , constitue une traduction française du Basic Empathy Scale (BES) développé par Jolliffe et Farrington (2006) pour les adolescents. L'instrument, administré aux enfants, mesure les composantes cognitive et affective de l'empathie. Les résultats d'empathie globale peuvent varier entre 20 (faible capacité d'empathie) et 100 (forte capacité d'empathie), la cotation des scores se faisant selon une échelle de 1 à 5 . La version française de l'instrument possède des qualités psychométriques satisfaisantes au niveau de la cohérence interne $(\alpha=$

2. Le GEM et le Social Stories Test ont été traduits selon la méthode de traduction/retraduction en utilisant une approche consensuelle. Cette méthode implique que deux personnes bilingues produisent chacune une traduction de façon indépendante, qu'elles comparent leurs versions respectives et résolvent les différences dans le texte, qu'une autre personne retraduise l'instrument dans sa langue d'origine afin de s'assurer que les énoncés ont conservé leur sens (Behling et Law, 2000), puis que la traduction soit révisée par un comité d'experts et d'utilisateurs potentiels de l'instrument (Bradley, 1994). La traduction n'a toutefois pas été validée. 
0,80 ) et la fidélité test-retest (intervalle de trois semaines) de l'échelle globale est acceptable $(r=0,66)$ (D'Ambrosio et al., 2009). L'indice de cohérence interne obtenu est satisfaisant $(\alpha=0,83)$.

Social Stories Test (SST; Sharp et al., 2007). Le questionnaire a été administré dans sa version française (Girard, Terradas et Achim, 2012b). II permet d'évaluer les biais de mentalisation chez les enfants d'âge scolaire et consiste en 15 vignettes, écrites et illustrées à l'aide de dessins, portant sur diverses situations sociales (p. ex., solitude, peur d'être ridiculisé, déménagement). Pour chaque vignette, trois choix de réponses sont présentés, qui permettent ensuite de classer ceux de l'enfant selon trois catégories. Deux de ces catégories sont liées à des attributions irréalistes et trop centrées sur l'enfant, soit des biais de mentalisation positifs ou négatifs, alors que la troisième catégorie concerne les attributions neutres, rationnelles et plutôt adaptatives. II existe deux versions de l'instrument, selon le sexe de l'enfant, afin de favoriser l'identification au personnage principal. La version anglaise du questionnaire comporte une bonne validité de construit, les biais de mentalisation étant positivement corrélés aux difficultés de comportement extériorisées chez les enfants d'âge scolaire (Sharp et al., 2007) ${ }^{3}$. Dans le cadre de la présente étude, les résultats ont permis d'établir quatre mesures : 1) nombre de biais de mentalisation négatifs rapportés, 2) nombre de biais de mentalisation positifs obtenus, 3) nombre de réponses neutres et 4) nombre de biais de mentalisation (positifs et négatifs) combinés. En raison de la petite taille de l'échantillon, les enfants n'ont pas été distribués dans des groupes mutuellement exclusifs selon le type de biais de mentalisation qu'ils présentent.

Entrevue d'attachement de l'enfant (Ensink et le groupe de recherche sur l'abus sexuel de l'Université Laval, 2002; d'après la version originale anglaise du Child Attachement Interview de Target et al., 1998; CAI). Cette entrevue semistructurée est composée de 15 questions qui permettent d'activer les relations d'attachement et de stimuler la production de récits d'attachement chez les enfants âgés de 7 à 12 ans. Bien que le CAI permette d'évaluer le style d'attachement et la CM de l'enfant, seule cette dernière a été évaluée dans le contexte de la présente recherche à l'aide de l'Échelle de fonctionnement réflexif de l'enfant (ÉFRE; Ensink, Target, Oandasan et Duval, 2015). La CM est ainsi mesurée à partir de sept questions du CAl évaluant l'habileté de l'enfant à aborder ses relations interpersonnelles et ses comportements en termes d'états mentaux (Ensink et al., 2014). Pour chaque réponse, l'enfant peut obtenir un score de -1 à 9 . Le score minimal $(-1)$ de cette échelle est associé à une $\mathrm{CM}$ négative, c'est-à-dire un refus de mentaliser ou une organisation bizarre et désintégrée de la personnalité (p. ex., l'enfant donne des explications inadéquates qui suggèrent la présence de difficultés quant à l'épreuve de réalité), alors que le score maximal (9) correspond à une CM exceptionnelle (Target, Oandasan et Ensink, 2001). Un score de 5 représente une $\mathrm{CM}$ se situant dans la moyenne. La CM globale de l'enfant correspond à la moyenne

3. La validation du SST ayant été effectuée à partir d'une analyse confirmatoire de classes latentes, les résultats sont complexes et difficiles à rapporter davantage dans l'espace disponible. Nous référons le lecteur à l'article publié par Sharp et al. (2007) cité dans la liste des références. 


\section{8}

statistique des scores obtenus à l'ensemble des questions. La fidélité inter-juges de l'échelle de cotation de la CM à partir du CAl est excellente (coefficients interclasses variant entre 0,80 et 0,90) et l'indice de cohérence interne est de 0,94 (Ensink et al., 2014). Dans le cadre la présente étude, trois assistantes de recherche formées et accréditées par K. Ensink ont coté la CM à partir du CAI. L'accord inter-juges pour $25 \%$ des protocoles cotés par les trois assistantes varie entre 0,74 et 0,98 , ce qui représente une forte corrélation. L'indice de cohérence interne est très satisfaisant $(\alpha=0,90)$.

\section{Résultats}

Des analyses descriptives ont d'abord été réalisées afin d'obtenir un portrait des participants sur l'ensemble des mesures d'empathie, de CM, de biais de mentalisation et de comportements extériorisés. Ensuite, en raison du petit échantillon ( $n=16)$, du fait que les postulats de normalité n'étaient pas rencontrés pour toutes les mesures, ainsi que de la présence de certaines données extrêmes, des analyses non-paramétriques ont été privilégiées.

\section{Description des participants}

Le Tableau 1 présente les moyennes, les écarts-types et les étendues des résultats des participants aux mesures d'empathie, de CM, de biais de mentalisation et de comportements extériorisés. En ce qui concerne les mesures d'empathie, les résultats des enfants indiquent une moyenne d'empathie globale de 56,63 (ÉT = $15,34)$, alors que les éducateurs rapportent une moyenne d'empathie globale de 6,19 (ÉT = 20,18). Afin de diminuer le nombre de variables incluses dans les analyses et étant donné la petite taille de l'échantillon, un score global d'empathie combinée a été créé en additionnant les scores standardisés obtenus aux questionnaires complétés par les enfants et les éducateurs. L'alpha de Cronbach global démontre la pertinence de ce choix $(0,85)$. Un test de rang signé de Wilcoxon d'échantillons associés comparant les scores globaux standardisés d'empathie des enfants tels qu'évalués par eux-mêmes et par les éducateurs a également été réalisé. Le résultat de ce test appuie la création du score global d'empathie combinée, alors qu'aucune différence significative n'est relevée entre les médianes pour les scores d'empathie rapportés par les enfants (Méd $=-0,20)(T=65 ; p=0,88 ; r=-0,03)$ et ceux rapportés par les éducateurs (Méd $=-0,03)$.

La CM globale des enfants, calculée à partir de la moyenne des scores obtenus aux sept questions de l'entrevue, est considérée répudiée (score de 0 ) ou absente (score de 1-2-3). Dans l'ensemble, les enfants présentent en moyenne davantage de réponses neutres $(8,13)$ que de biais de mentalisation positifs $(4,94)$ ou négatifs $(1,94)$. Les résultats des recherches précédentes concernant les liens entre les biais de mentalisation et les comportements extériorisés n'étant pas concluants et pouvant même être contradictoires (voir Girard et al., 2014, Ha et al,, 2011 et Sharp et al., 2007), nous avons opté pour créer un score global de biais de mentalisation. Ainsi, un score de biais de mentalisation combinés consistant en une addition des biais positifs et négatifs rapportés par chaque participant, a donc été créé afin de regrouper les deux types de biais de mentalisation présentés par 
chaque enfant. Finalement, tous les participants présentent des scores à la mesure de comportements extériorisés se situant dans la zone clinique.

Tableau 1. Moyennes, écarts-types et étendues de l'ensemble des variables

\begin{tabular}{lccc}
\hline Variables & Moyenne Écart-type & Étendue \\
\hline Empathie (enfants) & & & \\
Empathie cognitive & 30,25 & 8,61 & 16,00 à 45,00 \\
Empathie affective & 26,38 & 10,09 & 11,00 à 49,00 \\
Empathie globale & 56,63 & 15,34 & 34,00 à 94,00 \\
Empathie globale (score Z) & 0,00 & 1,00 & $-1,47$ à 2,44 \\
\hline Empathie (éducateurs) $^{2}$ & & & \\
Empathie cognitive & 1,19 & 6,04 & $-9,00$ à 11,00 \\
Empathie affective & 0,94 & 11,43 & $-20,00$ à 17,00 \\
Empathie globale & 6,19 & 20,18 & $-27,00$ à 40,00 \\
Empathie globale (score Z) & 0,00 & 1,00 & $-1,64$ à 1,68 \\
Empathie globale combinée & 62,81 & 30,80 & 10,00 à 134,00 \\
\hline Biais de mentalisation ${ }^{3}$ & & & 2,00 à 13,00 \\
Biais positifs & 4,94 & 2,77 & 1,00 à 10,00 \\
Biais négatifs & 1,94 & 2,24 & 0,00 à 7,00 \\
Absence de biais & 8,13 & 3,40 & 2,0 à 13,00 \\
Biais de mentalisation combinés & 6,88 & 3,40 & 2,00 \\
\hline Capacte & &
\end{tabular}

\section{Capacité de mentalisation ${ }^{4}$}

CM globale statistique

0,98

1,11

$-0,77$ à 2,88

\section{Comportements extériorisés ${ }^{5}$}

Comportements extériorisés (score T) $\quad 74,00 \quad 5,01 \quad 64,00$ à 85,00

Notes. ${ }^{1}$ Échelle d'empathie élémentaire : les scores varient entre 20 (faible capacité d'empathie) et 100 (forte capacité d'empathie). ${ }^{2}$ Mesure d'empathie de Griffith : les scores varient entre -92 (faible capacité d'empathie) et 92 (forte capacité d'empathie).

${ }^{3}$ Social Stories Test : il y a 15 vignettes qui comportent chacun trois choix de réponses, lesquelles se répartissent en biais neutre, biais de mentalisation positif ou biais de mentalisation négatif.

${ }^{4}$ Entrevue d'attachement de l'enfant : les scores varient entre -1 (mentalisation répudiée) et 9 (mentalisation exceptionnelle).

${ }^{5}$ Liste de vérification des comportements des jeunes de 6 à 19 ans : les scores varient entre 0 (absence de comportements extériorisés) et 100. 
Corrélations entre les variables à l'étude. Des analyses de corrélation ont été réalisées entre les scores d'empathie globale combinée, de biais de mentalisation combinés, de CM et de comportements extériorisés afin de vérifier la présence de liens entre ces variables (voir Tableau 2). Les résultats des analyses de corrélation bilatérale Tau-B de Kendall révèlent que les comportements extériorisés ne sont pas liés de façon significative à aucune autre mesure, que ce soit avec l'empathie globale combinée, la CM ou les biais de mentalisation combinés. Ainsi, aucun lien significatif n'est démontré entre les comportements extériorisés et la $\mathrm{CM}$, l'empathie ou les biais de mentalisation. Les corrélations effectuées indiquent toutefois une relation positive modérée entre le score global d'empathie combinée et ceux de l'échelle de $\mathrm{CM}$, une forte relation négative entre les résultats obtenus à l'échelle de $\mathrm{CM}$ globale et à l'échelle de biais de mentalisation combinés, ainsi qu'une relation négative modérée entre les résultats à l'échelle d'empathie globale combinée et à l'échelle de biais de mentalisation combinés.

Tableau 2. Corrélations bilatérales Tau-B de Kendall entre les comportements extériorisés, l'empathie globale combinée, la CM globale et les biais de mentalisation combinés

\begin{tabular}{lcccccc}
\hline & $\begin{array}{c}\text { Empathie globale } \\
\text { combinée }\end{array}$ & \multicolumn{2}{c}{ CM globale } & \multicolumn{3}{c}{$\begin{array}{c}\text { Biais de } \\
\text { mentalisation } \\
\text { combinés }\end{array}$} \\
\hline & $r$ & $p$ & $r$ & $p$ & $r$ & $p$ \\
\hline $\begin{array}{l}\text { Comportements } \\
\text { extériorisés }\end{array}$ & $-0,17$ & 0,365 & 0,09 & 0,618 & 0,07 & 0,715 \\
$\begin{array}{l}\text { Empathie globale } \\
\text { combinée } \\
\text { CM globale }\end{array}$ & & & 0,41 & 0,027 & $-0,34$ & 0,07 \\
\hline
\end{tabular}

Comparaison selon le service d'hébergement et le sexe des participants. Puisque les enfants évalués proviennent de deux unités du centre jeunesse, l'une interne et l'autre externe, une comparaison entre ces deux groupes de participants a été réalisée afin de vérifier s'il existe des différences significatives permettant de distinguer les participants en fonction de cette variable. Les résultats des analyses du test $U$ de Mann-Whitney pour échantillons indépendants ne révèlent aucune différence significative (voir le Tableau 3). Ainsi, les distributions pour toutes les mesures ne diffèrent pas significativement selon le service d'hébergement.

Afin de comparer les scores obtenus par les filles et les garçons de l'échantillon aux différentes mesures, un test $U$ de Mann-Whitney pour échantillons indépendants a été réalisé. Aucune différence significative n'est observée quant aux comportements extériorisés et aux biais de mentalisation combinés (voir le Tableau 4). Toutefois, les distributions des mesures de CM $(U=9,00 ; z=-2,10 ; p=$ $0,04 ; r=-0,52)$, d'empathie globale rapportée par les enfants $(U=3,50 ; z=-2,72$; $p=0,003 ; r=-0,68)$, d'empathie globale rapportée par les éducateurs $(U=10,00$; 
Tableau 3. Comparaison des médianes aux mesures d'empathie globale, de biais de mentalisation combinés, de $\mathrm{CM}$ globale et de comportements extériorisés selon les unités d'hébergement

\begin{tabular}{lccccc}
\hline & $\begin{array}{c}\text { Unité } \\
\text { interne } \\
(n=8)\end{array}$ & $\begin{array}{c}\text { Unité } \\
\text { externe } \\
(n=8)\end{array}$ & & & \\
\hline Variables & $M d n$ & $M d n$ & $U$ & $p$ & $r$ \\
\hline Empathie globale (enfants) & 54,50 & 53,50 & 31,00 & 0,96 & $-0,03$ \\
Empathie globale (éducateurs) & 2,50 & 12,00 & 40,00 & 0,44 & 0,21 \\
$\begin{array}{l}\text { Score global d'empathie } \\
\text { combinée }\end{array}$ & 57,00 & 69,00 & 36,00 & 0,72 & 0,11 \\
$\begin{array}{l}\text { Biais de mentalisation combinés } \\
\text { CM globale statistique }\end{array}$ & 7,00 & 5,00 & 22,00 & 0,33 & $-0,26$ \\
$\begin{array}{l}\text { Comportements extériorisés } \\
\text { (score T) }\end{array}$ & 0,85 & 1,38 & 40,50 & 0,38 & 0,22 \\
\hline
\end{tabular}

Tableau 4. Comparaison des médianes aux mesures d'empathie globale, de biais de mentalisation combinés, de $\mathrm{CM}$ globale et de comportements extériorisés selon le sexe des participants

\begin{tabular}{lccccc}
\hline & $\begin{array}{c}\text { Filles } \\
(n=5)\end{array}$ & $\begin{array}{c}\text { Garçons } \\
(n=11)\end{array}$ & & & \\
\hline Variables & $M d n$ & $M d n$ & $U$ & $p$ & $r$ \\
\hline Empathie globale (enfants) & 63,00 & 49,00 & 3,50 & 0,003 & $-0,68$ \\
Empathie globale (éducateurs) & 23,00 & 2,00 & 10,00 & 0,05 & $-0,50$ \\
$\begin{array}{l}\text { Score global d'empathie } \\
\text { combinée }\end{array}$ & 86,00 & 53,00 & 4,00 & 0,005 & $-0,67$ \\
$\begin{array}{l}\text { Biais de mentalisation combinés } \\
\text { CM globale statistique }\end{array}$ & 6,00 & 6,00 & 34,50 & 0,44 & 0,20 \\
$\begin{array}{l}\text { Comportements extériorisés } \\
\text { (score T) }\end{array}$ & 2,23 & 0,69 & 9,00 & 0,04 & $-0,52$ \\
\hline
\end{tabular}

$z=-2,00 ; p=0,05 ; r=-0,50)$ et d'empathie globale combinée $(U=4,00 ; z=-2,66$; $p=0,005 ; r=-0,67$ ) diffèrent significativement selon le sexe, les filles présentant des médianes plus élevées que celles des garçons.

\section{Discussion}

Les résultats de la présente étude démontrent que l'ensemble des participants présentent des scores cliniques à l'échelle de comportements extériorisés, ce qui concorde avec la tendance mise en lumière dans plusieurs autres études (p. ex., Finzi et al., 2001; Geoffrion et Ouellet, 2013; Hukkanen et al., 1999; Lawrence et al., 2006; Lefebvre, 2012) selon laquelle les comportements 


\section{2}

extériorisés sont très présents au sein de cette population. Pourtant, dans la présente étude, il n'y a pas de différence significative entre les garçons et les filles concernant la présence de comportements extériorisés. Ces résultats contredisent ceux d'autres recherches selon lesquelles les garçons sont surreprésentés dans ce type de problème comparativement aux filles (p. ex., Beaulac et Pageau, 2009). En effet, Sharp (2006) suggère que les filles ont tendance à manifester l'agressivité de façon subtile tandis que les garçons recourent davantage à des comportements extériorisés pour exprimer leur agressivité ou leur mal-être. Ainsi, les résultats d'une étude réalisée par Malti, Gummerum, Keller et Buchmann (2009) montrent que les filles font preuve d'un plus haut niveau de motivation au plan moral, de sympathie et de comportements pro-sociaux que les garçons. II demeure cependant que l'ensemble des enfants participant à la présente étude, autant les filles que les garçons, ont été exposés à différentes formes de maltraitance au sein de leur famille d'origine. À cet effet, Allen et al. (2008) suggèrent qu'en plus d'occasionner des traumas sévères, la violence augmenterait le risque que la victime s'engage ultérieurement à faire usage de violence envers autrui.

Aucune association significative entre les comportements extériorisés et les variables à l'étude, soit l'empathie globale combinée, la CM et les biais de mentalisation, n'a été démontrée, allant ainsi à l'encontre des résultats d'études antérieures ayant constaté la présence de tels liens chez les enfants ( $p$. ex., Girard et al., 2014; Ha et al., 2011; Ostler et al., 2010; Sharp, 2006; Sharp et al., 2007). À cet égard, dans leur recension critique des écrits scientifiques portant sur l'empathie et l'agressivité chez les enfants et les adolescents, Lovett et Sheffield (2007), ont montré que les liens entre ces deux variables étaient inconstants entre les études et que ceux-ci étaient davantage robustes chez les adolescents. Ainsi, une corrélation significative entre les comportements extériorisés et l'empathie peut être plus difficile à observer chez les enfants. Ceci peut être d'autant plus vrai que dans le cas de la présente recherche, la taille de l'échantillon était très petite. Par conséquent, il est possible que la puissance statistique n'ait pas été suffisante pour permettre de détecter un lien entre les variables à l'étude. Par ailleurs, l'échantillon était très homogène, puisque tous les participants présentent des scores cliniques à l'échelle de comportements extériorisés. Le même phénomène quant à l'homogénéité des résultats obtenus s'observe pour la CM et les biais de mentalisation. Ainsi, le manque de variance dans l'échantillon a pu rendre plus difficile la détection d'une corrélation significative entre les variables.

Une étude réalisée par Ensink et al. (2014) à partir du même instrument de mesure de la CM avait permis de démontrer que les enfants victimes d'abus sexuels présentent une CM qualifiée d'absente. Les résultats obtenus dans le cadre de la présente recherche suggèrent également que les enfants refusent de mentaliser ou présentent une absence de mentalisation, ce qui signifie qu'il est particulièrement difficile pour eux d'utiliser les états mentaux pour se comprendre et se décrire, ainsi que pour comprendre et décrire leurs relations avec leurs parents. En effet, la CM est la faculté de comprendre les comportements en fonction des états mentaux sous-jacents, c'est-à-dire à attribuer des pensées, des émotions et des intentions à soi et aux autres, permettant d'expliquer les comportements et les relations (Allen et al., 2008). Ainsi, les enfants évalués font fréquemment des descriptions comportementales ou physiques des situations interpersonnelles, sans attribuer 
d'états mentaux aux personnes évoquées. Ils sont souvent incapables de répondre aux questions posées et refusent parfois de le faire, se montrant alors hostiles envers l'assistante de recherche ou cherchant à détourner les questions d'entrevue (p. ex., vont se cacher sous une table, questionnent sur un autre sujet).

Les filles de l'échantillon obtiennent des résultats plus élevés que les garçons aux mesures de CM. L'étude de Taubner et Curth (2013) menée auprès d'adolescents issus de la population générale rapportait également une différence significative quant au score de CM entre les garçons et les filles, ces dernières obtenant des résultats plus élevés. Toutefois, comme il existe peu d'études mesurant la CM chez les enfants, notamment chez ceux en protection de l'enfance, il est encore difficile de savoir si cette différence révèle une tendance réelle dans la population d'enfants hébergés ou si elle résulte d'une particularité du petit échantillon de la présente recherche. D'autres études seront donc nécessaires pour reproduire les résultats obtenus. II est toutefois important de rappeler que les capacités observées chez les garçons et les filles de l'échantillon se situent toutes deux dans des catégories de $\mathrm{CM}$ reliées à un refus de mentaliser ou à une absence de mentalisation. Par conséquent, les capacités plus développées ici rapportées chez les filles demeurent relatives.

Les résultats obtenus aux mesures d'empathie suggèrent également que les filles démontrent des scores plus élevés que les garçons, et ce, à toutes les mesures (auto-rapportée, rapportée par les éducateurs, combinée). Ces résultats concordent avec ceux obtenus par D'Ambrosio et al. (2009), Čavojová, Sirota et Belovičová (2012) et Dadds et al. (2008) auprès d'enfants et d'adolescents issus de la population générale, où les filles obtiennent également des scores d'empathie significativement supérieurs à ceux des garçons. En ce qui a trait aux scores standardisés d'empathie globale obtenus aux deux instruments de mesure, ceux-ci ne diffèrent pas significativement, ce qui suggère que la perception des éducateurs et des enfants quant au niveau d'empathie de ces derniers est relativement semblable.

Les études de validation des instruments permettent de comparer les résultats obtenus aux deux mesures d'empathie à ceux d'enfants de la population générale, afin de qualifier la qualité de l'empathie en l'absence de normes pour les instruments utilisés. Ainsi, les résultats d'une étude de validation du BES en Slovaquie, menée auprès de 426 jeunes âgés entre 10 et 16 ans (49\% de filles; $M_{\text {âge }}=13,49 ; \dot{E} T=0,50$ ) issus de la population générale, indiquent que la moyenne d'empathie globale obtenue par les garçons est de 64,15 (É $T=8,41$ ), alors qu'elle est de 73,18 (ÉT = 8,70) pour les filles (Čavojová et al., 2012). Les résultats pour les filles du présent échantillon sont semblables, la moyenne globale obtenue par celles-ci étant de 71,00 (ÉT $=14,93)$. Toutefois, une différence semble présente entre les scores globaux des garçons, ceux de l'échantillon obtenant une moyenne globale de 50,09 (ÉT = 10,66). L'étude de validation du GEM (Dadds et al., 2008) auprès d'une population générale indique quant à elle que la moyenne obtenue par les garçons âgés entre 7 et 10 ans au score d'empathie globale est de 30,28 (ÉT = 21,89 ), alors qu'elle est de 39,78 (ÉT $=21,53$ ) pour les filles. Par conséquent, les moyennes de $0,09(E ́ T=19,86)$ et de 19,60 (ÉT =14,64), respectivement obtenues par les garçons et les filles de l'échantillon, semblent beaucoup plus faibles que 
celles des enfants issus de la population générale. II apparait donc que les scores obtenus par les participants de la présente étude aux mesures d'empathie révèlent des capacités globalement plus faibles que celles des enfants de la population générale, particulièrement lorsque l'empathie est mesurée par le biais du GEM, pouvant ainsi laisser présager un certain déficit au niveau de cette habileté.

Les analyses de corrélation suggèrent que le score d'empathie globale combinée est significativement associé avec la $\mathrm{CM}$, de sorte que les enfants qui présentent des scores plus élevés à la mesure globale combinée d'empathie présentent également des scores plus élevés de CM. Bien qu'à notre connaissance il n'existe aucune étude empirique ayant examiné les liens unissant ces deux variables chez les enfants, notamment ceux hébergés en réadaptation, les prémisses développementales communes, ainsi que les recoupements quant à leur utilité pour rendre compréhensible l'état affectif d'autrui (Dubé et al., 2018), soutiennent l'idée d'une association entre ces deux capacités. En effet, la CM et l'empathie font toutes deux appels à des habiletés considérées " cognitives » qui permettent de se représenter l'état affectif de l'autre et d'y attribuer un sens. Par conséquent, il est fort plausible que les enfants qui démontrent des capacités plus développées d'empathie fassent également preuve de meilleures capacités de mentalisation, puisque les deux nécessitent d'être capables de se représenter les états mentaux d'une autre personne. Selon Girard et al. (2014), une bonne capacité de mentalisation requiert de la flexibilité mentale pour que la personne soit en mesure d'élaborer ce qui pourrait expliquer le comportement d'autrui et ainsi aller au-delà de ce qui est manifeste afin d'établir des liens entre les représentations mentales (les états mentaux sous-jacents au comportement) et la réalité concrète (les manifestations comportementales).

II ressort également que les enfants de l'échantillon tendent à recourir davantage à des réponses neutres qu'à des biais de mentalisations positifs ou négatifs. Parmi les biais de mentalisation, tous les enfants rapportent au moins un biais positif, tandis que plus de la moitié d'entre eux ne présentent aucun, ou alors un seul, biais négatif. Ainsi, de manière générale, aucune tendance ne se dégage quant à un recours problématique à des biais de mentalisation chez les enfants au sein de l'échantillon. Aucune corrélation significative n'est d'ailleurs obtenue avec la mesure de comportements extériorisés. Ces résultats sont surprenants dans la mesure où les études précédentes ayant utilisé le même instrument de mesure des biais de mentalisation ont démontré une corrélation significative entre les comportements extériorisés et les biais de mentalisation positifs (Ha et al., 2011; Sharp et al., 2007) ou négatifs (Girard et al., 2014) dans des échantillons issus de la population générale. Des différences de méthodologie et de population peuvent peut-être expliquer les résultats. En effet, non seulement les trois études ont été menées auprès d'enfants issus de la population générale, mais l'une d'entre elles (Girard et al., 2014) a été menée seulement auprès de garçons. Par ailleurs, les mesures de comportements extériorisés sont différentes de celle choisie dans le cadre de cette étude, les trois autres recherches ayant eu recours au Strengths and Difficulties Questionnaire (Goodman, 1997), complété par les parents des enfants évalués. Par ailleurs, deux études (Ha et al., 2011; Sharp et al., 2007) ont réparti les enfants dans trois groupes, selon des analyses de classes latentes, divisant ainsi 
les participants dans trois catégories mutuellement exclusives correspondant à un style de mentalisation négatif, positif ou neutre, sur la base des résultats obtenus au questionnaire.

Les analyses de corrélation révèlent que la $\mathrm{CM}$ est négativement associée avec le score de biais de mentalisation combinés, de sorte que les enfants qui présentent le moins de biais de mentalisation (positifs ou négatifs) obtiennent des scores plus élevés de CM. Cette association fait du sens considérant que les enfants qui attribuent des états mentaux qui ne sont pas élaborés ou justifiés durant l'entrevue sur la $\mathrm{CM}$ se voient accorder de faibles scores à cette habileté. De même, un enfant qui tend à interpréter de façon biaisée les intentions des autres risque d'avoir de la difficulté à réfléchir et à rendre compte de façon claire et élaborée des états mentaux qui sous-tendent les comportements d'autrui. Ainsi, ces attributions d'intentions, que l'enfant ne parvient pas à expliquer ou à justifier, s'apparentent à la définition des biais de mentalisation et à l'idée d'une mentalisation déformée (Sharp et Venta, 2012). À notre connaissance, toutefois, aucune étude n'a comparé les biais de mentalisation et la CM, tels que mesurés respectivement par le SST (Sharp et al., 2007) et l'Entrevue d'attachement de l'enfant (Ensink et al., 2002; d'après la version originale anglaise de Target et al., 1998) et l'ÉFRE (Ensink et al., 2014).

Une corrélation négative a également été obtenue entre la mesure de biais de mentalisation combinée et l'échelle d'empathie globale combinée, de sorte que les enfants qui présentent des scores plus élevés aux mesures d'empathie rapportent moins de biais de mentalisation. Cette relation semble plausible dans la mesure où les enfants qui présentent des biais de mentalisation tendent à interpréter les intentions ou les comportements d'autrui d'une manière déformée (Sharp et Venta, 2012), alors que pour faire preuve d'empathie, il faut être en mesure de partager l'expérience émotionnelle de l'autre et de se représenter celle-ci, c'est-àdire d'interpréter de façon juste les causes de l'émotion perçue (Decety et Jackson, 2004). Toutefois, à notre connaissance, la seule autre étude ayant examiné les liens entre ces deux notions (Girard et al., 2014) n'a pas permis de démontrer l'existence d'une telle corrélation significative.

Finalement, aucune différence significative quant aux mesures d'empathie globale combinée, de biais de mentalisation combinés, de CM ou de comportements extériorisés n'est observée entre les enfants selon leur service d'hébergement. Ainsi, ces enfants, qu'ils bénéficient des services de réadaptation en unité de vie en internat ou en foyer de groupe externe, ne présentent pas de caractéristiques permettant de les différencier. À première vue, ce résultat pourrait s'expliquer par la petite taille de l'échantillon, qui a pu rendre impossible de détecter une éventuelle différence entre ces deux groupes. II est également possible qu'en pratique, bien que certains critères aient été établis par les différents centres jeunesse pour différencier les milieux d'hébergement, la répartition des enfants au sein des divers services de la protection de l'enfance soit influencée par les observations cliniques des éducateurs ou sur des considérations administratives (p. ex., selon les places disponibles). Ainsi, on peut penser que les enfants qui bénéficient de services de réadaptation, bien qu'ils soient hébergés dans des unités différentes (p. ex., à l'externe et à l'interne), présentent plusieurs caractéristiques semblables, notamment quant aux comportements extériorisés qu'ils démontrent. Toutefois, 
l'étude d'Ostler et al. (2010), réalisée auprès de 26 enfants hébergés en famille d'accueil, indique des résultats selon lesquels $50 \%$ des enfants présentent des scores limites ou cliniques à l'échelle de comportements extériorisés du CBCL. Ces résultats contrastent avec ceux de la présente recherche dans laquelle tous les enfants hébergés, qu'ils soient dans une unité de réadaptation ou dans un foyer de groupe externe, manifestent des comportements extériorisés.

\section{Limites de l'étude et pistes de recherche futures}

Les principales limites de l'étude sont reliées à sa méthodologie. En effet, le petit échantillon disponible, en plus de l'homogénéité ou de l'étendue observées quant à la distribution des résultats pour certaines variables, limite le potentiel de généralisation des résultats obtenus. Dans le cadre de recherches futures, il serait important d'avoir recours à un échantillon plus grand d'enfants hébergés en centre jeunesse, provenant notamment de divers services (p. ex., unité de vie interne, foyer de groupe externe, famille d'accueil), en plus de comparer les résultats à ceux d'enfants provenant de la population générale. Par ailleurs, comme certains auteurs ont démontré l'importance de tenir compte du nombre de facteurs de risque environnementaux auxquels les enfants sont exposés (MacKenzie, Kotch, Lee, Augsberger et Hutto, 2011), du type de mauvais traitements dont ils ont été victimes, de même que de la fréquence, de la durée, de la sévérité et de la combinaison de ceux-ci (Li et Godinet, 2014; Petrenko et al., 2012), il serait pertinent de considérer le motif de placement lors des analyses. Ceci permettrait notamment de vérifier la nature des liens existants entre les événements difficiles ou traumatiques vécus par les enfants avant leur arrivée en centre jeunesse et leur capacité d'empathie, leur $\mathrm{CM}$, leurs biais de mentalisation et la présence de comportements extériorisés.

Par ailleurs, le fait que les questionnaires administrés aux enfants aient été lus et complétés à voix haute en présence d'une assistante de recherche, et parfois de l'éducateur de suivi, a pu augmenter la probabilité que les enfants choisissent des réponses socialement acceptables lorsque des choix leur étaient fournis, notamment au SST. D'ailleurs, la traduction française de ce questionnaire n'a pas été validée avant son utilisation. De plus, les observations des assistantes de recherche durant l'administration de l'instrument laissent croire que deux éléments ont pu affecter les résultats rapportés. D'abord, la consigne d'administration, qui implique que l'enfant se mette à la place du personnage principal de la vignette présenté, a été difficile à comprendre et à suivre pour la plupart des participants. La deuxième observation concerne les choix de réponses du questionnaire. En effet, la réponse correspondant à l'absence de biais de mentalisation était souvent évidente parmi l'ensemble des choix présentés. Ainsi, les enfants ont pu donner des réponses socialement acceptables, ce qui peut affecter la validité des résultats obtenus et expliquer la prépondérance de réponses neutres, donc l'absence relative de biais de mentalisation.

Le recours à des questionnaires écrits a également pu affecter, dans une certaine mesure, la validité des résultats rapportés. En effet, malgré la lecture à voix haute, certains enfants ont eu besoin d'explications additionnelles afin de bien comprendre la nature des tâches ou des choix de réponses proposés. À l'avenir, il serait intéressant d'avoir davantage recours à des mesures observationnelles et 
à des instruments sous forme d'entrevue, permettant ainsi aux enfants de donner des réponses ouvertes, ou à des mises en situation réalisées sous la forme de jeux. Par ailleurs, considérant que les séances d'évaluation de la présente étude ont été filmées, une analyse davantage qualitative du profil des participants pourrait être réalisée dans le futur.

\section{Conclusion}

Malgré ces limites, cette recherche constitue, à notre connaissance, la première étude québécoise documentant l'empathie et la CM d'enfants hébergés en service de réadaptation en centre jeunesse. Elle permet ainsi de présenter un premier portrait des caractéristiques de ces enfants en mettant notamment en lumière une capacité d'empathie globale relativement faible, une CM considérée absente, peu de biais de mentalisation et la présence significative de comportements extériorisés chez tous les enfants. À défaut d'avoir démontré la présence de liens significatifs entre l'empathie, la CM, les biais de mentalisation et les comportements extériorisés, d'autres études seront nécessaires pour vérifier à nouveau ces relations. Par ailleurs, comme les résultats révèlent des déficits au niveau de l'empathie et de la CM, des interventions basées sur la mentalisation ou visant à développer les capacités d'empathie pourraient être utiles et pertinentes afin d'outiller les éducateurs travaillant auprès d'enfants en centre jeunesse.

\section{Références}

Achenbach, T. M. et Rescorla, L. A. (2001). Manual for ASEBA School-Age Forms \& Profiles. Burlington, VT: University of Vermont, Research Center for Children, Youth, \& Families.

Achim, J. et Terradas, M. M. (2015). Le travail clinique en contexte pédopsychiatrique : l'apport de la mentalisation à la pratique d'aujourd'hui. Filigrane, 24(2), 79-92.

Akhtar, N. et Bradley, E. J. (1991). Social information processing deficits of aggressive children: Present findings and implications for social skills training. Clinical Psychology Review, 11, 621644.

Allen, J. G., Fonagy, P. et Bateman, A. W. (2008). Mentalizing in Clinical Practice. Washington, DC: American Psychiatric Publishing.

Baron-Cohen, S. et Wheelwright, S. (2004). The empathy quotient: An investigation of adults with Asperger syndrome or high functioning autism, and normal sex differences. Journal of Autism and Developmental Disorders, 34(2), 163175.
Beaulac, S. et Pageau, D. (2009). Les élèves handicapés ou en difficulté d'adaptation ou d'apprentissage. Rapport présenté au Ministère de l'Éducation, du Loisir et du Sport. Direction de l'adaptation scolaire et des services complémentaires. Document inédit, Canada.

Behling, O. et Law, K. S. (2000). Translating questionnaires and other research instruments: Problems and solutions. Thousand Oaks, CA: Sage.

Bradley, C. (1994). Translation of questionnaires for use in different languages and cultures. Dans C. Bradley (dir.), Handbook of Psychology and Diabetes (p. 43-53). Amsterdam, PaysBas: Harwood Academic Publishers.

Camras, L. A., Grow, J. G. et Ribordy, S. C. (1983). Recognition of emotional expression by abused children. Journal of Clinical Child Psychology, 12(3), 325328.

Čavojová, V., Sirota, M., et Belovičová, Z. (2012). Slovak validation of the Basic Empathy Scale in pre-adolescents. Studia Psychologica, 54(3), 195-208. 
Centre jeunesse de la Montérégie. (2017). Nos services : services de réadaptation. Repéré à http://www. centrejeunessemonteregie.qc.ca/servi ces/services-de-readaptation/servicesde-readaptation-en-internat/

Centre jeunesse des Laurentides. (2017). Ressources intermédiaires. Repéré à http://www.cjlaurentides.qc.ca/nos-servi ces/hebergement-non-institutionnel/ ressources-intermediaires

Courtois, C. A. (2004). Complex trauma, complex reactions: Assessment and treatment. Psychotherapy: Theory, Research, Practice, Training, 41(2), 412-425.

Dadds, M. R., Hunter, K., Hawes, D. J., Frost, A. D. J., Vassallo, S., Bunn, P., ... et El Masry, Y. (2008). A measure of cognitive and affective empathy in children using parent ratings. Child Psychiatry and Human Development, 39, 111-122.

D'Ambrosio, F., Olivier, M., Didon, D. et Besche, C. (2009). The basic empathy scale: A french validation of a measure of empathy in youth. Personality and Individual Differences, 46(2), 160-165.

Decety, J. et Jackson, P. L. (2004). The functional architecture of human empathy. Behavioral and Cognitive Neuroscience Reviews, 3(2), 71-100. doi:10.1177/1534582304267187

Decety, J. et Jackson, P. L. (2006). A socialneuroscience perspective on empathy. Current Direction in Psychological Science, 15(2), 54-58.

de Vignemont, F. et Singer, T. (2006). The empathic brain: How, when and why? TRENDS in Cognitive Sciences, 10(10), 435-441. doi:10.1016/j.tics.2006.08.008

de Wied, M., Gispen-de Wied, C. et van Boxtel (2010). Empathy dysfunction in children and adolescents with disruptive behavior disorders. European Journal of Psychology, 626, 97-103.

Directeurs de la protection de la jeunesse. (2018). La cause des enfants tatouée sur le cœur. Bilan des directeurs de la protection de la jeunesse / directeurs provinciaux 2018. Repéré à https://www. ciusss-capitalenationale.gouv.qc.ca/ sites/default/files/dpj_bilan2018.pdf

Dodge, K. A. (1980). Social cognition and children's aggressive behavior. Child Development, 51, 162-170.

Domon-Archambault, V. et Terradas, M. M. (2015). Efficacité d'une formation fondée sur la mentalisation auprès des intervenants en centre jeunesse : étude pilote. Revue québécoise de psychologie, 36(3), 183-208.

Dubé, G., \& Terradas, M. M., \& DomonArchambault, V. (2018). Empathie, mentalisation et traumas complexes : quels liens avec les comportements extériorisés des enfants en protection de l'enfance? Enfance, 2, 343-372.

Ensink, K. et le groupe de recherche sur l'abus sexuel de l'Université Laval. (2002). Child Attachment Interview : version française. D'après la version originale anglaise de Target, M., Fonagy, P., Shmueli-Goetz, Y., Datta, A. et Schneider, T. (1998). The Child Attachment Interview (CAl) protocol, Revised Edition VI. Document inédit, Département de psychologie, Université Laval, QC.

Ensink, K., Normandin, L, Target, M., Fonagy, P., Sabourin, S. et Berthelot, N. (2014). Mentalization in children and mothers in the context of trauma: An initial study of the validity of the Child Reflective Functioning Scale. British Journal of Developmental Psychology, 33(2), 203217. doi :10.1111/bjdp.12074

Ensink, K., Target, M., Oandasan, C. et Duval, J. (2015). Child and Adolescent Reflective Functioning Scale (CRFS) Scoring Manual - for application with the Child Attachment Interview (CAI) (Age range 8-17 years). Document inédit, Département de psychologie, Université Laval, QC.

Finzi, R., Ram, A., Har-Even, D., Shnit, D. et Weizman, A. (2001). Attachment styles and aggression in physically abused and neglected children. Journal of Youth and Adolescence, 30(6), 769-786. 
Fonagy, P., Bateman, A. W. et Luyten, P. (2012). Introduction and Overview. Dans A. W. Bateman et P. Fonagy (dir.), Handbook of mentalizing in mental health practice (p. 3-42). Arlington, VA: American Psychiatric Publishing.

Fonagy, P. et Target, M. (1996). Playing with reality: I. Theory of mind and the normal development of psychic reality. The International Journal of Psychoanalysis, 77(2), 217-233.

Fonagy, P. et Target, M. (1997). Attachment and reflective function: Their role in self-organization. Development and Psychopathology, 9, 679-700. doi:10.1017/S0954579497001399

Fonagy, P. et Target, M. (2000). Playing with reality: III. The persistence of dual psychic reality in borderline patients. The International Journal of Psychoanalysis, 81(5), 853-873.

Fonagy, P. et Target, M. (2007). Playing with reality: IV. A theory of external reality rooted in intersubjectivity. The International Journal of Psychoanalysis, 88, 917-937. doi:10.1516/ijpa.2007.917

Geoffrion, S. et Ouellet, F. (2013). Quand la réadaptation blesse? Éducateurs victimes de violence. Criminologie, 46(2), 263-289. doi:10.7202/1020996ar

George, C., Kaplan, N. et Main, M. (1996). Adult Attachment Interview Protocol. Document inédit.

Girard, E., Terradas, M. M. et Achim, J. (2012a). Griffith Empathy Measure: traduction française. D'après la version originale de Dadds, M. R., Hunter, K., Hawes, D. J., Frost, A. D. J., Vassallo, S., Bunn, P., ... El Masry, Y. (2008). Document inédit, Département de psychologie, Université de Sherbrooke, QC.

Girard, E., Terradas, M. M. et Achim, J. (2012b). The Social stories test: traduction française. D'après la version originale de Sharp, C., Croudace, T. J. et Goodyer, I. M. (2007). Document inédit, Département de psychologie, Université de Sherbrooke, QC.
Girard, É., Terradas, M. M. et Matte-Gagné, C. (2014). Empathie, biais de mentalisation, comportements pro-sociaux et troubles de comportement chez les enfants d'âge scolaire. Enfance en difficulté, 3, 77-105. doi:10.7202/1028013ar

Goodman, R. (1997). The Strengths and Difficulties Questionnaire: A research note. Journal of Child Psychology and Psychiatry, 38, 581-586.

Greenson, R. R. (1977). Théorie et pratique de la psychanalyse. Paris, France : Presses Universitaires de France.

Ha, C., Sharp, C. et Goodyer, I. (2011). The role of child and parental mentalizing for the development of conduct problems over time. European Child and Adolescent Psychiatry, 20, 291-300. doi:10.1007/s00787-011-0174-4

Healey, C. V. et Fisher, P. A. (2011). Young children in foster care and the development of favorable outcomes. Children and Youth Services Review, 33, 1822-1830. doi:10.1016/j. childyouth.2011.05.007

Houzel, D. (2000). Empathie. Dans D. Houzel, M. Emmanuelli et F. Moggio (dir.), Dictionnaire de psychopathologie de l'enfant et de l'adolescent (p. 226). Paris, France : Presses Universitaires de France.

Hukkanen, R., Sourander, A., Bergroth, L. et Piha, J. (1999). Follow-up of children and adolescents in residential care in children's homes. Nordic Journal of Psychiatry, 53(3), 185-189.

Jolliffe, D. et Farrington, D. P. (2006). Development and validation of the Basic Empathy Scale. Journal of Adolescence, 29(4), 589-611.

Lawrence, C. R., Carlson, E. A. et Egeland, B. (2006). The impact of foster care on development. Development and Psychopathology, 18(1), 57-76. doi:10.1017/S0954579406060044 
Lefebvre, R. (2012). Tableaux supplémentaires sur les données principales de l'ECl-2008 : problèmes de fonctionnement chez l'enfant selon les principaux mauvais traitements corroborés et le risque de futurs mauvais traitements (Feuillet d'information 104). Repéré sur le site du Portail canadien de la recherche en protection de l'enfance : http://cwrp.ca/fr/infosheets/problemesfonctionnement-enfant-principauxmauvais-traitements-corrobores

LeSure-Lester, G. E. (2000). Relation between empathy and aggression and behavior compliance among abused group home youth. Child Psychiatry and Human Development, 31, 153-161.

Li, F. et Godinet, M. T. (2014). The impact of repeated maltreatment on behavioral trajectories from early childhood to early adolescence. Children and Youth Services Review, 36, 22-29.

Lovett, B. J. et Sheffield, R. A. (2007). Affective empathy deficits in aggressive children and adolescents: A critical review. Clinical Psychology Review, 27, 1-13. doi:10.1016/j.cpr.2006.03.003

Luke, N. et Banerjee, R. (2012). Maltreated children's social understanding and empathy: A preliminary exploration of foster carers' perspective. Journal of Child and Family Studies, 21, 237-246. doi:10.1007/s10826-011-9468-x

MacKenzie, M. J., Kotch, J. B., Lee, L.-C., Augsberger, A. et Hutto, N. (2011). A cumulative ecological-transactional risk model of child maltreatment and behavioral outcomes: Reconceptualizing early maltreatment report as risk factor. Children and Youth Services Review, 33, 2392-2398.

Malti, T., Gummerum, M., Keller, M. et Buchmann, M. (2009). Children's moral motivation, sympathy, and prosocial behavior. Child Development, 80(2), 442-460.

Miller, P. A. et Eisenberg, N. (1988). The relation of empathy to aggressive and externalizing/antisocial behavior. Psychological Bulletin, 103(3), 324-344.
Music, G. (2011). Trauma, helpfulness and selfishness: The effect of abuse and neglect on altruistic, moral and prosocial capacities. Journal of Child Psychotherapy, 37(2), 113-128. doi:10.1 080/0075417X.2011.581466

Nadeau, M.-E., Nolin, P. et Chartrand, C. (2013). Behavioral and emotional profiles of neglected children. Journal of Child \& Adolescent Trauma, 6(1), 11-24. doi:10.1080/19361521.2013.732202

Ostler, T., Bahar, O. S. et Jessee, A. (2010). Mentalization in children exposed to parental methamphetamine abuse: Relations to children's mental health and behavioral outcomes. Attachment \& Human Development, 12(3), 193-207. doi:10.1080/14616731003759666

Pears, K. C. et Fisher, P. A. (2005). Emotion understanding and theory of mind among maltreated children in foster care: Evidence of deficits. Development and Psychopathology, 17, 47-65. doi:10.1017/S0954579405050030

Petrenko, C. L. M., Friend, A., Garrido, E. F., Taussig, H. N. et Culhane, S. E. (2012). Does subtype matter? Assessing the effects of maltreatment on functioning in preadolescent youth in out-of-home care. Child Abuse \& Neglect, 36, 633644.

Rogosch, F. A., Cicchetti, D. et Aber, J. L. (1995). The role of child maltreatment in early deviations in cognitive and affective processing abilities and later peer relationship problems. Development and Psychopathology, 7(4), 591-609.

Sharp, C. (2006). Mentalizing problems in childhood disorders. Dans J. G. Allen et P. Fonagy (dir.), Handbook of mentalization-based treatment (p. 101121). Chichester, Angleterre: John Wiley \& Sons.

Sharp, C., Croudace, T. J. et Goodyer, I. M. (2007). Biased mentalizing in children aged seven to 11: Latent class confirmation of response styles to social scenarios and associations with psychopathology. Social Development, 16(1), 181-202. 
Sharp, C. et Venta, A. (2012). Mentalizing problems in children and adolescents. Dans N. Midgley et I. Vrouva (dir.), Minding the child. Mentalization-based interventions with children, young people and their families (p. 35-53). Hove, Angleterre: Routledge.

Sutton, J., Reeves, M. et Keogh, E. (2000). Disruptive behaviour, avoidance of responsibility and theory of mind. British Journal of Developmental Psychology, 18(1), 1-11. doi:10.1348/026151000165517

Target, M. et Fonagy, P. (1996). Playing with reality: II. The development of psychic reality from a theoretical perspective. The International Journal of PsychoAnalysis, 77(3), 459-479.

Target, M., Fonagy, P., Shmueli-Goetz, Y., Datta, A. et Schneider, T. (1998). The Child Attachment Interview (CAI) protocol, revised edition VI. Document inédit.

Target, M., Oandasan, C. et Ensink, K. (2001). Child reflective functioning scoring manual for application to the Child Attachment Interview. Document inédit.
Tarren-Sweeney, M. (2013). An investigation of complex attachment- and traumarelated symptomatology among children in foster and kinship care. Child Psychiatry and Human Development, 44(6), 727-741. doi:10.1007/s10578013-0366-X

Taubner, S. et Curth, C. (2013). Mentalization mediates the relation between early traumatic experiences and aggressive behavior in adolescence. Psihologija, 46(2), 177-192. doi:10.2298/ PSI1302177T

Taubner, S., White, L. O., Zimmerman, J., Fonagy, P. et Nolte, T. (2013). Attachment-related mentalization moderates the relationship between psychopathic traits and proactive aggression in adolescence. Journal of Abnormal Child Psychology, 41, 929938. doi:10.1007/s10802-013-9736-x 